\section{Naturally Acquired Sarcoptes scabiei Infestation in a Captive Southern Tamandua (Tamandua tetradactyla) and a Capybara (Hydrochoeris hydrochaeris)}

\section{Abstract}

A privately-owned southern tamandua (Tamandua tetradactyla) and a capybara (Hydrochoeris hydrochaeris) were presented for severe pruritus. Superficial skin scrapings revealed numerous Sarcoptes scabiei mites. Morphological examination and mitochondrial DNA sequencing revealed that both patients were infested with Sarcoptes scabiei. The animals were treated with selamectin $(9 \mathrm{mg} / \mathrm{kg}$ ) topically once, and the owner was instructed to repeat treatment in two weeks. The tamandua died of unknown causes three weeks after initial examination before reevaluation could occur. Clinical signs and skin lesions in the capybara resolved after two treatments with selamectin. This is the first documented report of sarcoptic mange in a southern tamandua. In addition, this is the first documented case of natural transmission of Sarcoptes mites between a tamandua and capybara in captivity. Finally, this is the first described use of topical selamectin for the treatment and resolution of sarcoptic mange in a capybara.

Keywords: Capybara; Hydrochoeris hydrochaeris; Lesser anteater; Selamectin; Scabies; Tamandua tetradactyla

Received: August 21, 2017; Accepted: August 23, 2017; Published: September 13, 2017

\section{Case Report}

Sarcoptes scabiei was first a human parasite that spread to various domestic and captive animal hosts, and later moved to certain wildlife species, especially canids, felids, and bovids [1]. Sarcoptes mites recovered from different hosts are indistinguishable morphologically, and genetic analyses show that the genus Sarcoptes contains a variable single species with several hostadapted strains [1]. Clinically, sarcoptic mange is characterized by severe pruritus, papules, alopecia, crusts, excoriations, and lichenification [2-9], which may become generalized in chronic cases, but typically, lesions concentrate at elbows, hocks, pinnal margins, and ventral abdomen [10]. Infestations are most common in crowded, unclean settings, where they are highly contagious. The mite is difficult to diagnose unless the animal is heavily infested, most tests lack sensitivity, and superficial skin scrapings may yield mites only $20 \%$ of the time [3].

The southern tamandua, or lesser anteater (Tamandua tetradactyla), is a forest-dwelling species native to South America [11] Common typical ectoparasites are ticks, psoroptid mites, and
Berger DJ ${ }^{1}$, Chelladurai JJ2, Brewer $\mathrm{MT}^{2}$, Mertins $\mathrm{JW}^{3}$, Zaffarano $\mathrm{BA}^{1}$ and Ratliff $\mathrm{CM}^{4 *}$

1 Department of Veterinary Clinical Sciences, lowa State University, 1800 Christensen Drive, Ames, IA 50011, USA

2 Department of Veterinary Pathology, Iowa State University, 1800 Christensen Drive, Ames, IA 50011, USA

3 United States Department of Agriculture, Animal and Plant Health Inspection Service, Veterinary Services, Science, Technology, and Analysis Services, National Veterinary Services Laboratories, 1920 Dayton Avenue, Ames, IA 50010, USA

4 University of Wisconsin-Madison School of Veterinary Medicine, 2015 Linden Drive, Madison, WI 53706, USA

* Corresponding author: Ratliff CM

झ cratliff@wisc.edu

University of Wisconsin-Madison School of Veterinary Medicine, 2015 Linden Drive, Madison, WI 53706, USA.

Tel: 4693966009

Citation: Berger DJ, Chelladurai JJ, Brewer MT, Mertins JW, Zaffarano BA, et al. (2017) Naturally Acquired Sarcoptes scabiei Infestation in a Captive Southern Tamandua (Tamandua tetradactyla) and a Capybara (Hydrochoeris hydrochaeris). J Vet Med Surg. Vol. 1 No. 3: 14.

fleas [12-14]. Tamanduas are frequently exhibited in zoos, and less commonly, owned privately as pets. The capybara (Hydrochoeris hydrochaeris) is a large rodent inhabiting riverbanks and marshlands in Central and South America. They also are common in zoos or privately owned for exhibition. At least 19 tick species 
infest wild capybaras, and captive animals commonly sustain sarcoptic mange $[2,7]$.

\section{Case 1}

A 3-year-old female intact southern tamandua presented for a 3-week history of pruritus and an exudative excoriation on the right flank. The animal was housed in a $2 \times 1 \times 2 \mathrm{~m}$ wire cage with aluminum flooring, bedded with pine shavings. The patient's cage was kept in a room shared with a capybara but these animals never contacted each other directly. On physical examination, multifocal crusts and scale were observed the base of the pinnae, axillae, elbows, and inguinal region. A $4 \times 4 \mathrm{~cm}$ erythematous excoriated patch on the right flank (Figure 1) exuded serosanguinous discharge centrally, with peripheral crusting. The interdigital spaces were severely erythemic and moist. Patchy hypotrichosis was noted in the interscapular space, over the right shoulder, and on the dorsal surface of the muzzle. The patient was observed to be pruritic during the examination. An impression smear was acquired from the right flank. Hair was plucked from multiple areas for trichogram, and a sample for dermatophyte fungal culture was acquired via the Mckensie toothbrush technique. A crust at the cranial margin of the excoriation was lifted and the area underneath swabbed for bacterial and fungal cultures. Skin scrapings were performed at multiple affected sites.

Skin cytology revealed neutrophilic inflammation, with intra and extracellular coccoid and rod-shaped bacteria. No parasites or fungal agents were observed on trichogram, and dermatophyte test media revealed no growth after one month. Bacterial culture revealed heavy mixed growth of several species, including Staphylococcus aureus, Proteus mirabilis, Corynebacterium spp., and two species of Streptococcus, while fungal culture yielded a heavy growth of Candida albicans. Skin scrapings taken from the left elbow and right inguinal fold revealed S. scabiei mites.

Ceftiofur (ceftiofur sodium, Naxcel, Pfizer, New York, New York 10017, USA; 3 mg/kg i.m., b.i.d. for three days) was prescribed

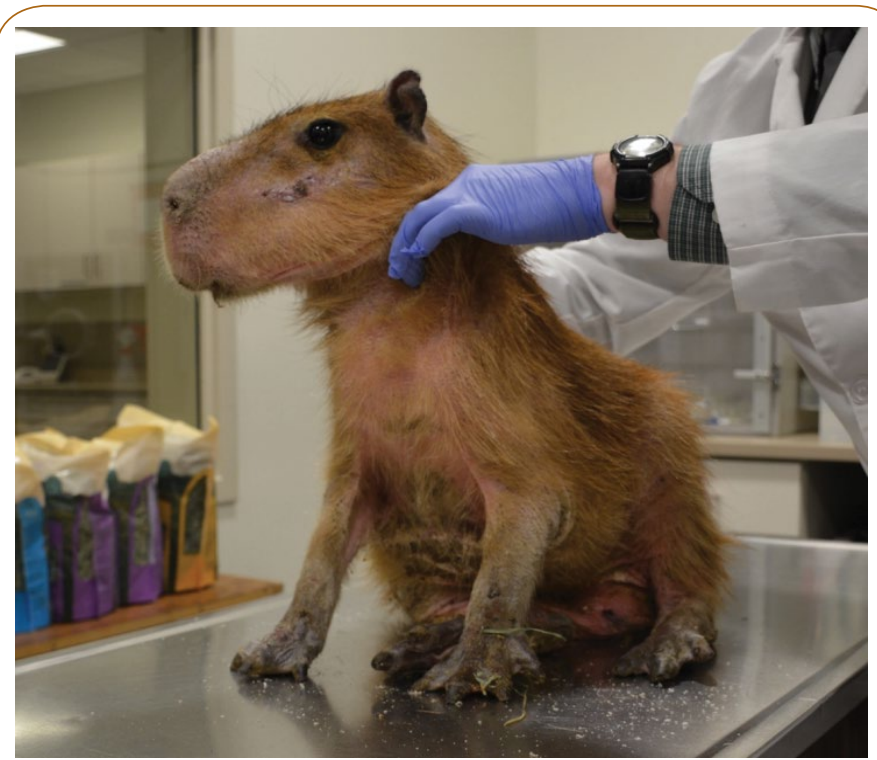

Figure 1

Clinical photograph, Case 1. Sarcoptic mange; excoriation and crusting along the right flank of a southern tamandua (Tamandua tetradactyla). for treatment of presumed opportunistic bacterial skin infection. Selamectin (Revolution, Zoetis Inc., Kalamazoo, Michigan 49007, USA; $9 \mathrm{mg} / \mathrm{kg}$ topically, once), was applied between the shoulders, with an additional dose for owner-applied retreatment in two weeks. Repeat skin scraping was planned four weeks after initial examination, but the tamandua died due to unknown causes three weeks after evaluation. A necropsy was performed, but poor post-mortem preservation of the body allowed marked autolysis and prevented accurate determination of death.

\section{Case 2}

A 3-year-old intact female capybara presented for pruritus of two weeks duration. This patient was owned and housed as described in Case 1, but it was examined six weeks later. On examination, the capybara was diffusely erythemic with generalized scale. Multifocal crusts and patches of hyperkeratosis were present along the venter, with excoriations on the face and flanks. The patient was emaciated and had mucopurulent nasal discharge (Figure 2). Skin scrapings from the ventral chest, left periocular region, and left elbow yielded numerous live suspected S. scabiei. Subsequent standard tissue maceration, flotation, and microscopic examination of slide-mounted mites (Figure 3) confirmed the identification. Other skin scrapings were collected for molecular analysis. The capybara was treated with selamectin (Zoetis; $9 \mathrm{mg} / \mathrm{kg}$ topically, once) and the owner repeated treatment in two weeks. The capybara also was treated for respiratory disease with enrofloxacin (Baytril, Bayer Animal Health, Shawnee Mission, Kansas 66216, USA; 15 mg/kg p.o., b.i.d. for two weeks). There was no follow-up appointment, but per client telephone communication three months later, pruritus and skin lesions resolved after two selamectin treatments, and the patient remained symptom-free.

DNA was extracted from skin scrapings obtained from both hosts, using the DNeasy Blood and Tissue Kit (QIAGEN Inc., Valencia, California 91355, USA) and following manufacturer's protocol. DNA was eluted in $200 \mu \mathrm{L}$ of water and stored at $-20^{\circ} \mathrm{C}$. 700 base pairs of the mitochondrial cytochrome $C$ oxidase subunit 1 (Cox1) were amplified in $50 \mu \mathrm{L}$ reactions, using $2 \mu \mathrm{L}$ of DNA, $3 \mathrm{mmol}$

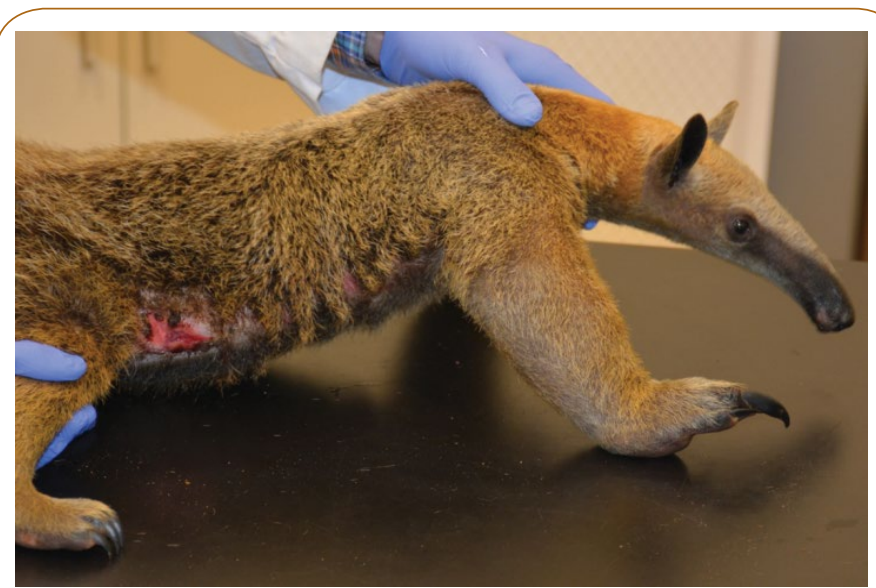

Figure 2 Clinical photograph, Case 2. Sarcoptic mange; diffuse erythema, hypotrichosis to alopecia, with scaling and focal crusting along the face, distal extremities, and ventral surfaces of a capybara (Hydrochoeris hydrochaeris). 


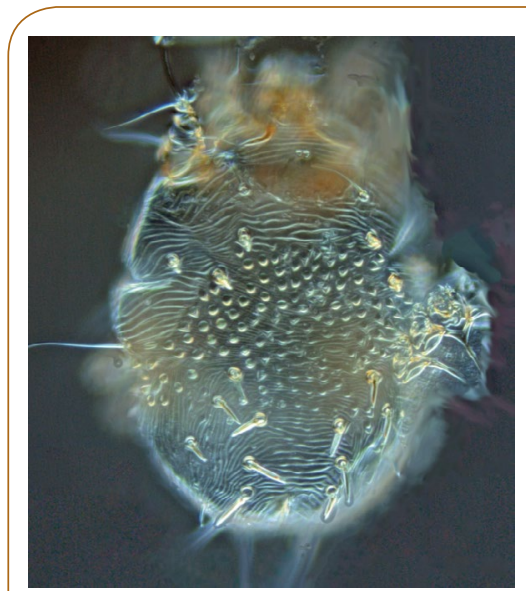

Figure 3 Photomicrograph, Case 2. Female Sarcoptes scabie mite, isolated from skin scraping from the capybara and showing diagnostic striations, setae, and spines on the dorsal cuticle. Potassium hydroxide maceration, slidemounted in Sheather's solution, differential interference contrast illumination, X250.

of $\mathrm{MgCl}_{2}, 200 \mu \mathrm{mol}$ of dNTPs, $1 \times$ PCR buffer, 2 units of Taq DNA Polymerase (Promega Corp., Madison, Wisconsin 53704, USA), and $2.5 \mu \mathrm{mol}$ each of primers $5^{\prime}$-GACACCCAGAAGTTTACATTC-3' and 5'-TATATTTTGATAATGAATCTC-3' [6]. Thermocycling conditions with denaturation at $94^{\circ} \mathrm{C}$ for $2 \mathrm{~min}, 5$ cycles of $94^{\circ} \mathrm{C}$ for $15 \mathrm{~s}, 45^{\circ} \mathrm{C}$ for $15 \mathrm{~s}, 72^{\circ} \mathrm{C}$ for $1 \mathrm{~min}$, followed by 30 cycles of $94^{\circ} \mathrm{C}$ for $15 \mathrm{~s}, 50^{\circ} \mathrm{C}$ for $15 \mathrm{~s}, 72^{\circ} \mathrm{C}$ for $1 \mathrm{~min}$, and a final extension step of $72^{\circ} \mathrm{C}$ for $5 \mathrm{~min}$.

Presence of amplification products was confirmed with agarose gel electrophoresis on $1.5 \%$ gels. Products were purified using the Wizard SV Gel and PCR Clean-Up System (Promega). Sequencing was performed on an Applied Biosystems 3730xl DNA Analyzer. Trimming and pairwise sequence alignments were conducted using MEGA6.06 [13]. Nucleotide BLAST searches were performed to compare sequences with those in NCBI GenBank. Sequences were deposited in GenBank (Banklt1952082 CapCox $1^{\circ} \mathrm{KX} 827305$, AntCox1 KX827306). Partial Cox1 sequences obtained after sequencing were trimmed to $543 \mathrm{bp}$. Pairwise sequence alignment of the partial Cox1 gene of Sarcoptes derived from both hosts revealed $100 \%$ mutual identity. BLAST results revealed that the sequences had $99.63 \%$ identity with S. scabiei types suis and canis; $99.44 \%$ identity with S. scabiei types vulpes, wallaby and wombati; $99.26 \%$ identity with S. scabiei types hominis and oryctolagus 2; and $99.08 \%$ identity with S. scabiei type oryctolagus.

This report describes the first documented case of sarcoptic mange in any anteater and the evident transmission of mites between a captive capybara and a tamandua. Despite unsubstantiated generic mentions of $S$. scabiei from anteaters in two review papers, $[4,8]$ there are no previous, verified primary reports of sarcoptic mange in any member of the Vermilingua, although the mites do infest certain other pilosans [12].

Microscopy and genetic sequencing confirmed that the mites recovered from both patients were $S$. scabiei. The source of these mites remains unknown, but they were genetically identical between the two patients, indicating transmission among these exotic hosts or infestation from a common source. Considering that individual strains of S. scabiei are highly host adapted, transmission from one exotic species to another completely unrelated species is unusual. The genetic similarity between the mites recovered from these cases and those posted to GenBank are most compatible the canid or suiid type mite, although the sequences are not $100 \%$ identical. Both patients were part of a collection used in a traveling show for entertainment and educational purposes, but neither had been exhibited at any venues for months prior to diagnosis. Both patients had resided with the owner for two years, and their origin was not disclosed.

The efficacy of selamectin for treatment of sarcoptic mange in the tamandua is undetermined because the animal died before reexamination. However, rare mites were seen on the skin during necropsy. The owner did not report any adverse effects such as vomiting, diarrhea, ataxia or seizures prior to the tamandua being found deceased. The presence of renal tubular degeneration and hemorrhage found within the gastrointestinal tract could represent an unrelated cause of death or be due to autolysis post-mortem, but neither is consistent with reported selamectin toxicity. Although post-treatment skin scrapings for the capybara were not performed, client communication indicated complete clinical resolution following two doses of selamectin applied topically two weeks apart.

Sarcoptes scabiei is capable of infesting both the capybara and Southern tamandua, resulting in pruritus and classical lesions associated with sarcoptic mange. Circumstances suggest that these mites can be transmitted between proximately housed host species, and they may have further zoonotic potential. Topical treatment with selamectin may provide a viable treatment option in future cases.

\section{References}

1 Bornstein S, Morner T, Samuel WM (2001) Sarcoptes scabiei and sarcoptic mange, Parasitic disease of wild mammals. 2nd edn. Ames (IA): lowa State University Press, UK.

2 Corriale MJ, Orozco MM, Jiménez PI (2013) Population parameters and sanitary status of capybaras (Hydrochoerus hydrochaeris) in artificial lagoons of Esteros del Iberá. Neotrop Mammal 20: 31-45.

3 Curtis CF (2012) BSAVA manual of canine and feline dermatology. 3rd edn. Gloucester (UK): British Small Animal Veterinary Association, UK.

4 Diniz LSM (2001) Biology, medicine and surgery of South American wild animals. Ames (IA): lowa State University Press, USA.

5 Diniz LSM, Costa EO, Oliveira PMA (1995) Clinical disorders observed in anteaters (Myrmecophagidae, Edentata) in captivity. Vet Res Commun 19: 409-415.

6 Erster O, Roth A, Pozzi PS, Bouznach A, Shkap V (2015) First detection of Sarcoptes scabiei from domesticated pig (Sus scrofa) and genetic characterization of $S$. scabiei from pet, farm and wild hosts in Israel. Exp Appl Acarol 66: 605-612.

7 García SGG, Castro AM, Rodríguez I, Bermúdez CSE (2014) Ixodid ticks of Hydrochoerus isthmius Goldman, 1912 (Rodentia: Caviidae) in Panama. Syst Appl Acarol 19: 404-408. 
8 Gillespie D (2011) Xenarthra: Edentata (anteaters, armadillos, sloths). In: Fowler ME, Miller RE (eds.), Zoo and wild animal medicine. 5th edn. St. Louis (MO): WB Saunders Co, USA.

9 Hayssen V(2011) Tamandua tetradactyla (Pilosa: Myrmecophagidae). Mammal Spec 43: 64-74.

10 Miller Jr WH, Griffin CE, Campbell KL (2013) Muller and Kirk's small animal dermatology. 7th edn. St. Louis (MO): Saunders-Elsevier, USA.

11 The IUCN Red List of Threatened Species (2017) Available from: http://www.iucnredlist.org/details/21350/0
12 Sibaja-Morales KD, de Oliveira JB, Rocha JAE, Gamboa HJ, Gamboa PJ, et al. (2009) Gastrointestinal parasites and ectoparasites of Bradypus variegatus and Choloepus hoffmanni sloths in captivity from Costa Rica. J Zoo Wild Med 40: 86-90.

13 Tamura K, Stecher G, Peterson D, Filipski A, Kumar S (2013) MEGA6: Molecular Evolutionary Genetics Analysis version 6.0. Mol Biol Evol 30: 2725-2729.

14 Whitaker Jr JO, Abrell DB (1987) Notes on some ectoparasites from mammals of Paraguay. Entomol News 98: 198-204. 